Ältere Patientinnen mit Ovarialkarzinom

\section{Chemotherapie nicht vorenthalten}

Zweifel zum Einsatz einer Chemotherapie bei älteren Patientinnen sind nicht angebracht, wie eine Metaanalyse von drei Phase-II/III-Studien der Nordostdeutschen Gesellschaft für Gynäkologische Onkologie zeigt. Insgesamt 1.213 Patientinnen mit rezidiviertem Ovarialkarzinom hatten an diesen Studien teilgenommen. Das mediane Alter zum Zeitpunkt der Diagnose betrug 59 Jahre. 349 Patientinnen waren $\geq 65$ Jahre alt, 864 Patienten $<65$ Jahre.

Hämatologische Toxizitäten traten häufiger bei Patientinnen über 65 Jahren auf (Odds Ratio 1,35; p = 0,028). Vor allem Anämien, Leukopenien und Thrombozytopenien waren mit einem höheren Alter assoziiert. Dagegen entwickelten beide Altersgruppen vergleichbar häufig nicht hä- matologische Toxizitäten ( $\mathrm{p}=0,901)$. Hinsichtlich der Zahl verabreichter Chemotherapiezyklen und notwendiger Dosisreduktionen gab es keinen signifikanten Unterschied zwischen den Gruppen. Auch Therapieabbrüche waren bei älteren Patientinnen nicht häufiger als bei jüngeren.

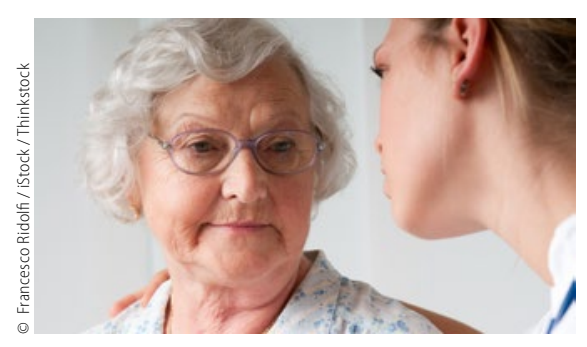

Das Alter allein ist kein Grund, auf eine Chemotherapie zu verzichten.
Nach der Adjustierung für kardiovaskuläre Erkrankungen und Diabetes waren bei älteren Patientinnen das progressionsfreie Überleben (Hazard Ratio [HR] 1,20; $\mathrm{p}=0,01)$ und auch das mediane Gesamtüberleben signifikant kürzer (HR 1,$21 ; \mathrm{p}=0,021$ ).

Also kommen hämatologische Nebenwirkungen der Chemotherapie bei Patientinnen über 65 Jahren zwar häufiger vor als bei jüngeren. Aber diese haben keinen Einfluss auf die Häufigkeit von Therapieabbrüchen. Deshalb sollte älteren Patientinnen eine adäquate Chemotherapie allein wegen ihres Alters nicht vorenthalten werden. In dieser Patientinnengruppe sind ein sorgfältiges geriatrisches Assessment und Monitoring erforderlich.

Judith Neumaier

Woopen $\mathrm{H}$ et al. Elderly ovarian cancer patients: An individual participant data meta-analysis of the North-Eastern German Society of Gynecological Oncology (NOGGO). Eur J Cancer. 2016;60:101-6.

Standard bei fortgeschrittenem Nierenzellkarzinom?

\section{Gute Daten für neuen Tyrosinkinaseinhibitor}

Cabozantinib ist ein oraler Inhibitor von Tyrosinkinasen wie MET, VEGFR und AXL. In der offenen, randomisierten Phase-III-Studie METEOR wurde Cabozantinib bei Patienten mit fortgeschrittenem oder metastasiertem Nierenzellkarzinom und Progression nach Vorbehandlung mit einem VEGFR-Tyrosinkinaseinhibitor (TKI) mit dem mTOR-Inhibitor Everolimus verglichen. Es zeigten sich unter dem TKI ein längeres Gesamtüberleben, eine spätere Krankheitsprogression und ein besseres objektives Ansprechen als unter Everolimus. 658 Patienten hatten randomisiert einmal täglich $60 \mathrm{mg}$ Cabozantinib oder $10 \mathrm{mg}$ Everolimus erhalten.

Primärer Endpunkt war das progressionsfreie Überleben (PFS). Zum Zeitpunkt der geplanten Auswertung war das PFS in der Cabozantinib-Gruppe signifikant länger als in der Everolimus-Gruppe (median 7,4 vs. 3,9 Monate; Hazard Ratio [HR] 0,51, 95\%-Konfidenzintervall [95\%-KI] 0,41-0,62; $<<0,0001)$.

Die finalen Ergebnisse zum Gesamtüberleben (OS) basieren auf einer nicht geplanten, zweiten Zwischenauswertung. 18,7 Monate betrug die mediane Beobach- tungsdauer bis zu dieser Auswertung in der Cabozantinib-Gruppe, 18,8 Monate in der Everolimus-Gruppe. Das mediane OS lag bei 21,4 versus 16,5 Monaten (HR 0,66, $95 \%$-KI 0,53-0,83; p = 0,00026). Die Therapie mit Cabozantinib führte zudem deutlich häufiger zu einem objektiven Ansprechen ( 17 vs. $3 \%$; $\mathrm{p}<0,0001$ ).

\section{kurz notiert}

In allen untersuchten Subgruppen war der klinische Nutzen von Cabozantinib zu beobachten. Das Sicherheitsprofil entsprach den bisherigen Beobachtungen.

Aufgrund dieser Ergebnisse sollte Cabozantinib als neue Standardtherapie für diese Patienten betrachtet werden, so die Forscher.

Judith Neumaier

Choueiri TK et al. Cabozantinib versus everolimus in advanced renal cell carcinoma (METEOR): final results from a randomised, open-label, phase 3 trial. Lancet Oncol. 2016;17(7):917-27.

\section{Bakterielle Nanoroboter für die Krebstherapie}

Ein kleines Magnetfeld reicht, dann dringt Magnetococcus marinus tief in den Tumor ein, vor allem in hypoxische Bereiche, die sonst therapeutisch kaum zugänglich sind. Dort können die Bakterien präzise ihre Last abladen, z. B. Medikamente. Die Bakterien orientieren sich an Magnetfeldern - und das lässt sich nutzen, um ihre Bewegung zu manipulieren. In ersten Tierversuchen schienen die bakteriellen Nanoroboter ihre Aufgabe gut zu erfüllen. Kanadische Forscher injizierten Magnetococcus peritumoral in immundefiziente Mäuse mit transplantierten Tumoren. Dreidimensionale Magnetfelder leicht über der Erdmagnetfeldstärke lockten die Bakterien zuverlässig in den Tumor. Wie spezielle Färbetechniken und elektronenmikroskopische Aufnahmen zeigten, versammelten sich dort die Keime tatsächlich in den hypoxischen Zonen, [Felfoul O et al. Nat Nanotechnol. 2016;Aug 15. (Epub ahead of print)]. Durch den aktiven Transport ließen sich bisher nicht erreichte Carrier-Konzentrationen im Tumorinneren erreichen. Als nächstes beluden die Wissenschaftler ihre biologischen Nanoroboter mit Liposomen. Damit gelangten sie ebenso gut wie ohne Last in den Tumor. Nach Auffassung der Forscher könnten auf diese Weise deutlich höhere Arzneimittelkonzentrationen im Tumorinneren erreicht werden als mit bisherigen Verfahren - ohne dass der Körper darunter mehr zu leiden hätte. Thomas Müller 